\title{
Conhecimento sobre HIV/aids segundo idosos de cidades da Serra Gaúcha/RS
}

\author{
Knowledge about HIV/aids by elderly people in southern Brazil cities \\ Bruna Capellari $^{1}$ (D), Amanda Oss Vidor ${ }^{1}$ (D), Marina Ferri Pezzini² (D), Stefany Godoy ${ }^{1}$ (D), Virgínia Maffei ${ }^{1}$ (D), \\ Julia Poeta ${ }^{3}$ (D)
}

\section{RESUMO}

Objetivo: Devido à carência de pesquisas nessa área, este estudo buscou avaliar o conhecimento sobre HIV/aids dos participantes dos grupos de convivência de cidades da Serra Gaúcha - RS. Métodos: O estudo foi delineado do tipo transversal (prevalência) e a amostra foi coletada por conveniência. Foram convidados a participar todos os integrantes de grupos de convivência de idosos de cidades da Serra Gaúcha - RS, com idade igual ou superior a 60 anos, que aceitaram participar da pesquisa, respondendo totalmente o questionário e assinando o termo de consentimento livre e esclarecido. Foi aplicado o questionário validado QHIV3I com questões relativas ao HIV/AIDS. As questões estão organizadas nos domínios "conceito", "transmissão", "prevenção", "vulnerabilidade", "diagnóstico" e "tratamento". Resultados: Participaram do estudo 647 idosos, sendo a maioria do sexo feminino $(73,4 \%)$ com idade entre 60 a 92 anos. Mais da metade dos participantes possuía renda familiar de até três salários mínimos (89,8\%). Dos entrevistados, $78,7 \%$ afirmaram ter como grau de escolaridade de um a sete anos e $74 \%$ sabiam que o HIV é o causador da AIDS. Em relação à transmissão, salienta-se que 23,8\% afirmaram que o vírus pode ser transmitido por sabonetes, toIhas e assentos sanitários e 39,3\% mencionaram que o vírus pode ser transmitido pelo mosquito. Quanto à prevenção, $87,3 \%$ declararam não utilizar camisinha nas relações sexuais e $88,2 \%$ nunca realizaram o teste para detecção do HIV. Conclusão: Constatou-se que ainda há dúvidas a respeito desse assunto, demonstrando a necessidade de campanhas direcionadas à população idosa, constituindo um desafio para a saúde pública.

Palavras-chave: HIV; Idoso; Conhecimento.

\begin{abstract}
Objective: Due to the lower number of studies in this area, this analysis aims to evaluate the HIV knowledge regarding elderly coexistence groups of Serra Gaúcha region in Rio Grande do Sul - Brazil. Methods: The study is characterized as transversal (prevalence). Participants of elderly coexistence groups in the cities of Serra Gaúcha/ RS aged 60 years old or more were welcomed to participate, answering completely the questionnaire and signed the consent. We used a validated questionnaire about HIV/AIDS, established in the areas "concept", "transmission", "prevention", "vulnerability", "diagnosis", and "treatment." Results: Taking a sample of 647 elderly subjects, the majority being female (73.4\%), and aged between 60-92, over half of the participants had a family income of one to three minimum wages (89.8\%), $78.7 \%$ declared having low education level (zero to seven years of study), and $74 \%$ knew that AIDS is caused by HIV. About the transmission, we noted that $23.8 \%$ said that the virus can be transmitted by towels, soaps, and toilet seats, and $39.3 \%$ reported the virus can be transmitted by mosquito. For the prevention, $87.3 \%$ reported not using condoms during sexual relations, and $88,2 \%$ never took the HIV detection test. Conclusion: As a result, there are still doubts and a lack of knowledge about the topic, regarding the subjects investigated, demonstrating the need for targeted campaigns to the elderly population, which may be a great challenge for public health.
\end{abstract}

Keywords: HIV; Elderly; Knowledge.

1. Biomédica. Centro Universitário da Serra Gaúcha (FSG), Caxias do Sul (RS), Brasil.

2. Mestre. Programa de Pós-Graduação em Ciências da Gastroenterologia / Hepatologia, Universidade Federal do Rio Grande do Sul (UFRGS), Porto Alegre (RS), Brasil.

3. Docente. Instituto Ciências da Saúde, Centro Universitário Ritter dos Reis (UniRitter), Porto Alegre (RS), Brasil.

$\bowtie$ Marina Ferri Pezzini. Hospital de Clínicas de Porto Alegre (HCPA). Rua Ramiro Barcelos, 2350 - Santa Cecília. CEP: 90035-903. Porto Alegre (RS), Brasil. pezzinifm@gmail.com | Recebido: 02/04//2019 | Aprovado:06/08/2019 


\section{INTRODUÇÃO}

O processo de envelhecimento populacional observado em todos os continentes é um fato marcante para a sociedade atual ${ }^{1}$. O aumento do número de idosos está impondo mudanças profundas nos modos de pensar e viver a velhice na sociedade. Nesse contexto, a saúde aparece como elemento central exercendo forte impacto sobre a qualidade de vida $^{2,3}$.

De acordo com a OMS (Organização Mundial de Saúde), é considerado idoso, o indivíduo com idade maior ou igual a 60 anos, para os países em desenvolvimento, e 65 anos para os países desenvolvidos ${ }^{1}$. Segundo projeções estatísticas da OMS, no período de 1950 a 2025, o grupo de idosos no país deverá ter aumentado em quinze vezes, enquanto a população total em cinco. Assim, o Brasil ocupará o sexto lugar quanto ao contingente de idosos, alcançando, em 2025, cerca de 32 milhões de pessoas com 60 anos ou mais ${ }^{1,4}$. De acordo com estimativas do IBGE, em 2027, o RS teria um número de idosos superior ao número de crianças ${ }^{4}$.

Pelos dados da OMS, estima-se que havia 36,9 milhões de pessoas no mundo vivendo com HIV contabilizadas até o ano de 2017. Na América, os valores se aproximam de 3,3 milhões $^{5}$, sendo que $1 / 3$ dessa população vive no Brasil. Ou seja, aproximadamente 882.810 casos de AIDS foram identificados no país, de 1980 até 2017. Segundo dados do Boletim Epidemiológico AIDS/HIV de 2017, Porto Alegre lidera o ranking das capitais brasileiras com a maior taxa de detecção, apresentando 65,9 casos a cada 100.000 habitantes $^{6}$.

Apesar de inicialmente associada a adultos jovens, houve um aumento no número de pessoas com diagnóstico de HIV no Brasil, na faixa etária acima de 60 anos. Esse aumento tem sido denominado de leve "envelhecimento" resultando na mais nova característica da epidemia ${ }^{6,7}$. Enquanto na primeira fase da epidemia (1980/1997) foram notificados no Brasil apenas 2.191 homens e 656 mulheres com HIV com idade superior a 60 anos, segundo o Boletim Epidemiológico de HIV e DST de 2013; de 1998 a 2012, o número de casos nessa faixa etária cresceu sistematicamente, chegando a 9.225 homens e 4.936 mulheres contaminados ${ }^{5}$. No Estado do RS, a notificação para esse grupo etário é de 883 casos até janeiro de $2008^{8}$.
O crescente aumento do número de casos de HIV na população idosa no Brasil tem sido associado a diversos fatores, tais como: envelhecimento da população brasileira, maiores recursos financeiros para o acesso a profissionais do sexo, fácil acesso a medicamentos para distúrbios eréteis (fator que tem prolongado a atividade sexual) e a desmitificação do sexo na terceira idade. Portanto, a vivência da sexualidade juntamente com o atraso de políticas de prevenção direcionadas a este grupo etário têm tornado os idosos mais vulneráveis às doenças sexualmente transmissíveis (DSTs) ${ }^{7,9,10,11}$. A dificuldade do diagnóstico de infecção por HIV em pessoas com mais de 60 anos de idade é considerada muito relevante nesse caso $^{2,12}$.

Portanto, devido à carência de estudos na área, essa investigação procura verificar o grau de conhecimento sobre HIV/AIDS nos indivíduos dos grupos de convivência da terceira idade em cidades da Serra do Rio Grande do Sul.

\section{METODOLOGIA}

Este estudo caracteriza-se por ser do tipo transversal, sendo a amostra coletada por conveniência e composta por integrantes de grupos de convivência de idosos de cidades da Serra Gaúcha/RS. Foram incluídos no estudo os indivíduos com idade igual ou superior a 60 anos, que aceitaram participar da pesquisa, respondendo totalmente um questionário e assinando o termo de consentimento Livre e Esclarecido (TCLE). A amostragem por conveniência é frequentemente utilizada para a geração de ideias em pesquisas exploratórias, com a vantagem de permitir que a escolha de amostras e a coleta de dados sejam relativamente fáceis, porém, com a necessidade da elaboração e estruturação criteriosa de seleção, a fim de evitar possíveis vieses ${ }^{13}$. Nesta pesquisa, todos os representantes dos grupos de convivência das cidades citadas foram informados da pesquisa e concordaram em participar. Sendo assim, na data previamente marcada, o preenchimento do questionário foi agendado com os representantes de cada grupo e obtido via amostragem consecutiva e consentimento informado durante os dias e horários das reuniões. Este estudo foi 
aprovado pelo Comitê de Ética em Pesquisa Círculo/FSG de acordo com o parecer No 671.660.

Foi aplicado o questionário validado QHIV3I (modificado de Lazzarotto et al. ${ }^{14}$ ) com 16 questões relativas ao HIV/AIDS. Primeiramente, os participantes foram questionados sobre dados sociodemográficos que incluíram, sexo, idade, renda familiar, tempo de estudo, presença de parceiro fixo e religião praticante. As demais questões relativas ao conhecimento sobre o HIV/AIDS estão organizadas nos domínios "conceito", "transmissão", "prevenção", "vulnerabilidade", "diagnóstico" e "tratamento". Para demonstração do comportamento que define a vulnerabilidade em relação à contaminação pelo HIV, foi questionado o uso frequente de camisinha e a frequência de sexo com parceiros variáveis. A avaliação da confiabilidade foi executada com a utilização do coeficiente Kappa, que mediu o grau de concordância entre as respostas fornecidas nos dois momentos da pesquisa. Os resultados desse teste evidenciaram uma boa confiabilidade do QHIV3I, a ser corroborada por estudos posteriores. Os questionários foram aplicados no período de setembro de 2017 a julho de 2018, nos locais frequentados pelos grupos, seguindo um pré-agendamento de data e horário com seus representantes.

Os dados levantados pelo questionário foram consolidados no programa Microsoft Office Excel 2010, que foi utilizado também para a elaboração de tabelas e figuras. A análise foi realizada pelo IBM-SPSS V.18.0, por meio de estatísticas descritivas como: frequências, percentuais, médias e desvio padrão.

\section{RESULTADOS}

Dos 711 idosos convidados a participar do estudo, 55 deles recusaram-se a responder 0 questionário e 9 foram excluídos pois responderam de forma incompleta. Portanto, 647 foram incluídos na pesquisa, sendo todos moradores de alguma das treze cidades da Serra Gaúcha (Tabela 1).

A maioria dessa população era do sexo feminino $(73,4 \%)$, com média de idade $69,7 \pm 7$ anos. Os dados demográficos dos participantes estão apresentados na Tabela 2.

\section{Tabela 1}

Número de participantes do estudo descrito por cidades da região da Serra Gaúcha/RS.

\begin{tabular}{lrr}
\hline Cidade & $\mathrm{N}$ & $\%$ \\
\hline Nova Prata & 120 & 18,5 \\
Garibaldi & 24 & 3,7 \\
Nova Petrópolis & 16 & 2,5 \\
Veranópolis & 14 & 2,2 \\
Antônio Prado & 53 & 8,1 \\
Vila Flores & 14 & 2,2 \\
Nova Roma do Sul & 11 & 1,7 \\
Protásio Alves & 18 & 2,8 \\
Flores da Cunha & 103 & 15,9 \\
Salvador do Sul & 24 & 3,7 \\
Caxias do Sul & 191 & 30 \\
Bento Gonçalves & 33 & 5,1 \\
Farroupilha & 26 & 4,0 \\
Total & 647 & 100 \\
\hline
\end{tabular}

Fonte: próprio autor

\section{Tabela 2}

Características gerais dos participantes do estudo $(n=647)$

\begin{tabular}{|c|c|c|}
\hline $\begin{array}{l}\text { Parâmetros } \\
\text { sociodemográficos }\end{array}$ & $\%$ & Frequência \\
\hline \multicolumn{3}{|l|}{ Sexo } \\
\hline Masculino & 26,6 & 172 \\
\hline Feminino & 73,4 & 475 \\
\hline \multicolumn{3}{|l|}{ Escolaridade } \\
\hline Nenhuma & 3,7 & 24 \\
\hline 1 a 3 anos de estudo & 30,7 & 199 \\
\hline 4 a 7 anos de estudo & 48 & 310 \\
\hline 8 ou mais anos de estudo & 17,6 & 114 \\
\hline \multicolumn{3}{|l|}{ Renda mensal } \\
\hline Até 1 salário mínimo & 40,6 & 263 \\
\hline 1 a 3 salários mínimos & 49,2 & 318 \\
\hline 4 a 6 salários mínimos & 7,6 & 49 \\
\hline Mais de 7 salários & 2,6 & 17 \\
\hline \multicolumn{3}{|l|}{ Religião } \\
\hline Católica & 89,6 & 580 \\
\hline Outras & 4,8 & 31 \\
\hline Nenhuma & 5,6 & 36 \\
\hline \multicolumn{3}{|l|}{ Companheiro } \\
\hline Não & 42,7 & 276 \\
\hline Sim & 57,1 & 370 \\
\hline
\end{tabular}

Fonte: próprio autor 
Na amostra estudada, 74\% afirmaram que o HIV é o causador da AIDS; $82 \%$ que o vírus é identificado através de exames laboratoriais; e $41,4 \%$ disseram que indivíduos com anti-HIV positivo sempre apresentarão sintomas da doença. Em relação à transmissão, 23,8\% afirmaram que $o$ vírus pode ser transmitido por sabonetes, tolhas e assentos sanitários; $33,1 \%$ acreditam que abraço, beijo no rosto e beber no mesmo recipiente também são formas de transmissão; e, ainda, 39,3\% mencionaram que o vírus pode ser transmitido por picada de mosquito. Os resultados referentes a esses domínios estão descritos na Tabela 3.

Tabela 3

Conhecimentos gerais sobre HIV/AIDS dos participantes do estudo $(n=647)$

\begin{tabular}{|c|c|c|}
\hline Variáveis & Frequência & $\%$ \\
\hline \multicolumn{3}{|c|}{ Domínio "conceito" } \\
\hline \multicolumn{3}{|c|}{ O vírus HIV é o causador da AIDS? } \\
\hline Verdadeiro & 479 & 74 \\
\hline Falso & 33 & 5,1 \\
\hline Não sei & 135 & 20,9 \\
\hline \multicolumn{3}{|c|}{ A pessoa com o vírus da AIDS sempre apresenta os sintomas da doença? } \\
\hline Verdadeiro & 268 & 41,4 \\
\hline Falso & 178 & 27,5 \\
\hline Não sei & 201 & 31,1 \\
\hline \multicolumn{3}{|c|}{ O vírus da AIDS é identificado através de exames de laboratório? } \\
\hline Verdadeiro & 530 & 82 \\
\hline Falso & 26 & 4 \\
\hline Não sei & 91 & 14 \\
\hline \multicolumn{3}{|c|}{ Domínio "transmissão" } \\
\hline \multicolumn{3}{|c|}{ O vírus da AIDS pode ser transmitido por sabonetes, toalhas e assentos sanitários? } \\
\hline Verdadeiro & 154 & 23,8 \\
\hline Falso & 340 & 52,6 \\
\hline Não sei & 153 & 23,6 \\
\hline \multicolumn{3}{|c|}{$\begin{array}{l}\text { O vírus da AIDS pode ser transmitido por abraço, beijo no rosto, beber no mesmo copo e } \\
\text { chimarrão? }\end{array}$} \\
\hline Verdadeiro & 214 & 33,1 \\
\hline Falso & 319 & 49,3 \\
\hline Não sei & 114 & 17,6 \\
\hline \multicolumn{3}{|c|}{ O vírus da AIDS pode ser transmitido por picada de mosquito? } \\
\hline Verdadeiro & 254 & 39,3 \\
\hline Falso & 223 & 34,4 \\
\hline Não sei & 170 & 26,3 \\
\hline \multicolumn{3}{|c|}{ Domínio "prevenção" } \\
\hline \multicolumn{3}{|c|}{ A pessoa que usa camisinha nas relações sexuais impede a transmissão do vírus da AIDS? } \\
\hline Verdadeiro & 484 & 74,8 \\
\hline Falso & 74 & 11,4 \\
\hline Não sei & 89 & 13,8 \\
\hline \multicolumn{3}{|c|}{ Domínio "prevenção" } \\
\hline \multicolumn{3}{|c|}{ Existe uma camisinha específica para mulheres? } \\
\hline Verdadeiro & 384 & 59,3 \\
\hline Falso & 46 & 7,1 \\
\hline Não sei & 217 & 33,6 \\
\hline \multicolumn{3}{|c|}{ O uso da mesma seringa e agulha por diversas pessoas transmite AIDS? } \\
\hline Verdadeiro & 567 & 87,6 \\
\hline Falso & 31 & 4,7 \\
\hline Não sei & 49 & 7,7 \\
\hline
\end{tabular}


Tabela 3. (Continuação)

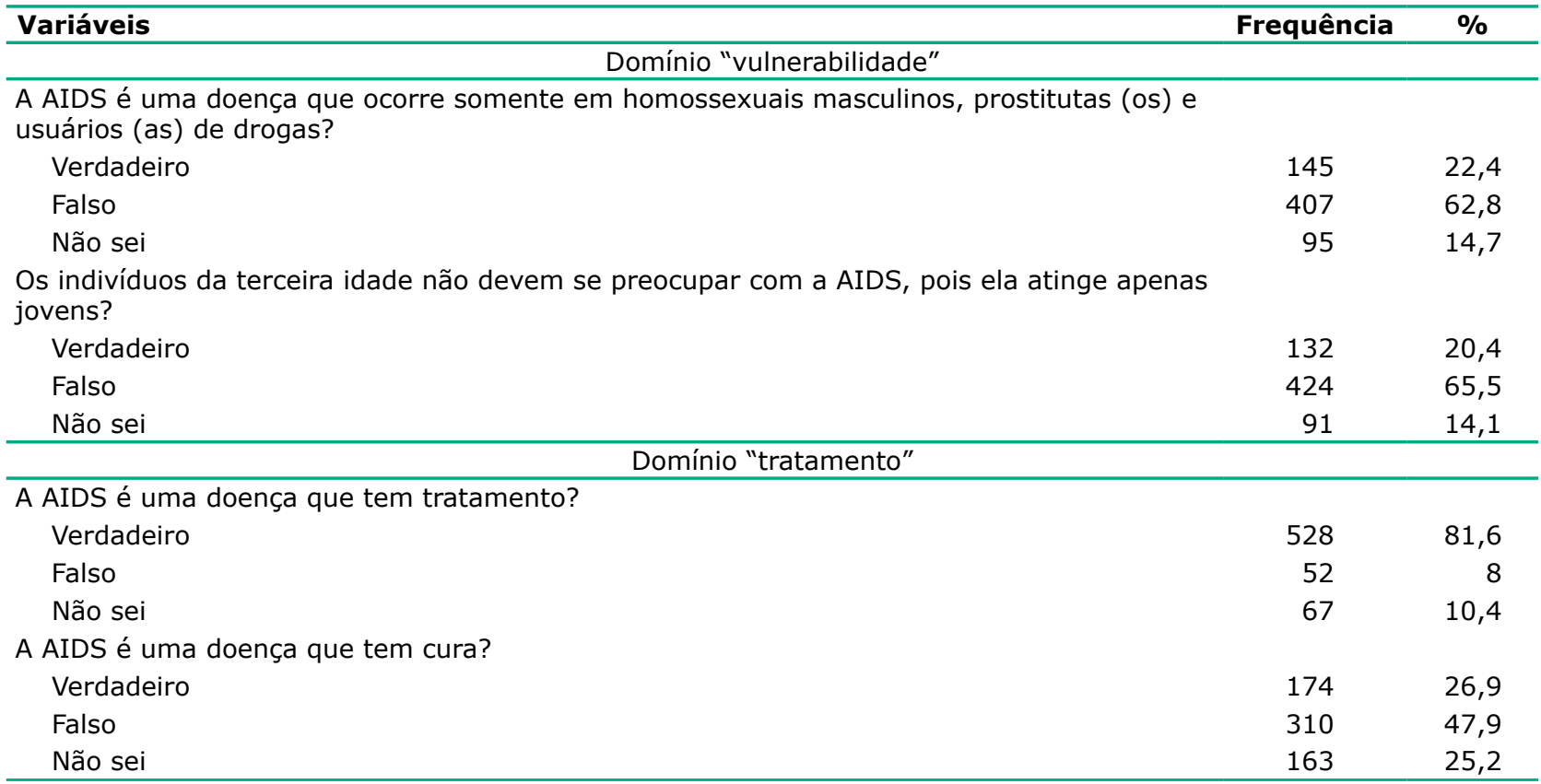

Fonte: próprio autor

Do total de entrevistados, $74,8 \%$ afirmaram que 0 uso do preservativo impede a transmissão do vírus, porém $87,3 \%$ não o utilizam em relações sexuais. Quando questionados sobre o teste para deteç̧ão do vírus HIV, $88,2 \%$ relataram nunca ter realizado, mesmo que $47,9 \%$ afirmaram ter conhecimento da AIDS se tratar de uma doença sem cura.

\section{DISCUSSÃO}

Nesta pesquisa, os idosos de ambos os sexos dos grupos de convivência das cidades visitadas da Serra Gaúcha responderam às questões organizadas em domínios. A representação feminina foi de $73,4 \%$, enquanto a masculina de $26,6 \%$. Estudos recentes demonstraram que as mulheres possuem uma sobrevivência superior aos homens de 5 anos $^{15,16,17}$. Isso também pode ser explicado pela proporção de 100 idosas para 82 idosos segundo fonte do IBGE (Instituto Brasileiro de Geografia e Estatística $)^{4}$.

Quando questionados a respeito do domínio "conceito", a maioria afirmou que o HIV é o responsável pela AIDS. Esse resultado é semelhante ao obtido por Camargo, Torres e Biazus ${ }^{17}$ nas cidades de Florianópolis, Rio do Sul, Porto Alegre e Erechim, nas quais $87,5 \%$ dos participantes concordaram que a AIDS é uma doença que surge a partir da infecção pelo vírus HIV. Esse vírus ataca o sistema imunológico de forma progressiva e gradativa, sendo as células de defesa T CD4+ o principal alvo ${ }^{2}$.

Neste estudo, 41,4\% expuseram que o indivíduo com o vírus sempre apresentará sintomas da doença. Tal resultado está de acordo com as respostas dos idosos da região do Vale do Rio dos Sinos, a partir do estudo realizado por Lazarotto et al. $(2008)^{14 .}$ Já Melo et al. (2012) ${ }^{18}$ questionou idosos e adultos jovens de Pernambuco, mapeando que $36,7 \%$ dos idosos discordaram quanto à possibilidade de uma pessoa com aparência saudável estar vivendo com HIV/AIDS, contrapondo-se aos adultos jovens que admitiram essa possibilidade $(98,4 \%)$. Portanto, evidenciou-se que ainda existe um estereótipo característico para as pessoas infectadas e que os indivíduos da terceira idade conhecem apenas a fase sintomática da doença.

Em relação ao conhecimento sobre o domínio "transmissão", grande parte dos indivíduos $(87,6 \%)$ acredita que o uso da mesma seringa e agulha por diversas pessoas transmite o HIV, porém quando indagados sobre a transmissão do vírus por sabonetes, toalhas e assentos sanitários, e outras questões referentes a abraço, beijo no rosto, 
compartilhamento de copos ou da cuia de chimarrão, a porcentagem de respostas incorretas foi preocupante $(23,8 \%$ e $33,1 \%$, respectivamente).

Segundo estudos realizados ${ }^{13,19}$, ao serem indagados sobre 0 fato do mosquito ser o transmissor do HIV, $79,9 \%$ e $41,4 \%$ dos participantes, respectivamente, afirmaram ser possível contrair a doença através do inseto. Da mesma maneira, no presente estudo, 39,3\% fizeram a mesma afirmação e $26,3 \%$ não souberam responder, ou seja, houve concordância de opiniões sobre a transmissão do vírus através da picada do inseto. Desde $1985^{20}$ e $1986^{21}$, sabe-se que o mosquito não pode ser considerado vetor na transmissão do HIV. Os principais fatores são a ausência do antígeno T4 na superfície celular dos artrópodes (impedindo, dessa forma, a sua replicação no mosquito), a baixa infectividade e a curta sobrevivência do vírus no mosquito ${ }^{22}$. Diante desses resultados, fica evidente que ainda há uma visão equivocada dos idosos a respeito da transmissibilidade do HIV, o que pode contribuir ainda mais para o preconceito devido à falta de conhecimento.

Sabe-se que apesar de todas as pesquisas desenvolvidas até o momento não existe cura para a AIDS, e o tratamento para doença inclui antirretrovirais que inibem a replicação do vírus, limitando o seu avanço22. Dentre os entrevistados, $81,6 \%$ conheciam sobre a existência de tratamento da AIDS e 47,9\% expuseram que a mesma não tem cura. Houve semelhança nesses dados com o estudo feito por Costa et al. $(2012)^{23}$ com idosos de Pernambuco. Com isso, nos dois estudos, metade da população, aproximadamente, não tinha o conhecimento do HIV causar uma doença sem cura, dado que deveria preocupar a saúde pública.

Quanto à prevenção, apesar de $74,8 \%$ dos participantes afirmarem que o uso do preservativo nas relações sexuais impede a transmissão do vírus da AIDS, , 87,3\% não o utilizam durante a relação. No estudo realizado por Costa et al. (2012) ${ }^{23}$, esse dado também é alarmante, pois $90 \%$ dos participantes não fazem uso do preservativo, constituindo um dos dados mais preocupantes. Uma provável explicação é a predominância das mulheres na menopausa, cuja gravidez indesejada deixa de ser uma questão preocupante, e o uso do preservativo, no entanto, torna-se pouco relevante. Por ou- tro lado, os idosos do sexo masculino têm dificuldade de utilizar o preservativo, por não saberem como usar, ou por terem medo de perder a ereção, transformando, dessa maneira, a população idosa em um grupo de risco, contribuindo para a transmissão do vírus e de outras DSTs ${ }^{9,19,24,25}$.

O aumento da expectativa de vida torna a prática de sexo sem proteção uma grande preocupação em termos de saúde pública em relação ao envelhecimento bem-sucedido. Essa prática pode ser determinada por diversas condições positivas de natureza biopsicossocial, cultural, espiritual, sendo evidenciada por um estado de saúde pleno ${ }^{12,26}$.

A situação abordada sobre a realização do teste de HIV apontou que $88,2 \%$ dos entrevistados nunca realizaram o teste, e esse fato alarmante talvez possa ser explicado pelo próprio preconceito de realizar o teste, ou pelo fato de que grande parte dos profissionais da saúde não considera os idosos como sexualmente ativos e vulneráveis à infecção pelo HIV, contribuindo para o diagnóstico tardio da doença.

O presente trabalho foi conduzido no sentido de verificar o conhecimento por grupo de idosos de cidades da Serra Gaúcha em relação ao HIV/AIDS. Em face a algumas respostas pouco satisfatórias, ficou evidente que ainda existem dúvidas a respeito desse assunto e principalmente em relação às formas de transmissão do vírus. $O$ fato de a maioria dos indivíduos não utilizar preservativo durante as relações sexuais demonstra que a população estudada se encontra em risco em relação ao HIV/ AIDS, bem como a outras DSTs.

Os resultados apresentados são de extrema importância, pois destacam a falta de conhecimento dos indivíduos da terceira idade da região pesquisada no que tange ao HIV/AIDS. Com o aumento da expectativa de vida e o envelhecimento saudável, torna-se fundamental a ampliação de campanhas direcionadas à população idosa, constituindo um desafio à saúde pública. Com campanhas mais específicas para essa faixa etária, os indivíduos serão capazes de adotar práticas seguras, a fim de evitar a infecção e reverter a tendência de aumento das DST e da AIDS. Os centros de convivência cujo objetivo principal é a valorização desse grupo, favorecendo a inclusão social, tornam-se excelentes locais para o desenvolvimento dessas campanhas, contanto que recebam o apoio de políticas públicas de prevenção. 
Nosso estudo possui algumas limitações, tais como ser realizado em apenas algumas cidades da Serra Gaúcha, podendo ter sido expandido para mais localidades, o que tonaria os resultados mais representativos e de maior significância. A amostra foi coletada por conveniência, não havendo um cálculo amostral prévio justificando o número de indivíduos incluídos. De fato, os resultados desse estudo servem de base para a elaboração de novos projetos com o intuito de incluir uma amostra baseada em cálculo amostral.

\section{REFERÊNCIAS}

1. UNAIDS, Joint United Nations Program on HIV/AIDS (UNAIDS). Global report: UNAIDS report on the global AIDS pandemic and World Health Organization (WHO) 2010. Disponível em: http://www.unaids.org/sites/ default/files/media_asset/20101123_globalreport_ en_1.pdf Acesso em 11 de junho de 2018.

2. Vieira RS, Viera RS. Saúde do idoso e execução da política nacional da pessoa idosa nas ações realizadas na atenção básica à saúde. Rev Direito Sanit [internet]. 2016 [acesso em 29 set 2019];17(1):14-37. Disponível em: http:// www.revistas.usp.br/rdisan/article/view/117042/114640

3. Kramer AS, Lazzarotto AR, Sprinz E, Manfroi WC. Alterações metabólicas, terapia antirretroviral e doença cardiovascular em idosos portadores de HIV. Arq Bras Cardiol [Internet]. 2009 [acesso em 18 out 2019];93(5):519-23. Disponível em: http://www.scielo.br/pdf/abc/v93n5/a19v93n5.pdf

4. IBGE, Censo demográfico 2010: Distribuição da população por idade e sexo. Resultados do universo, 2010.

5. http://www.who.int/en/news-room/fact-sheets/detail/ hiv-aids Acesso em 13 julho 2018.

6. BRASIL/Ministério da Saúde. Disponível em http://www. aids.gov.br/pt-br/pub/2018/boletim-epidemiologicohivaids-2018. Acesso em 17 dezembro 2018.

7. Santos AFM, Assis M. Vulnerabilidade das idosas ao HIV/ AIDS: despertar das políticas públicas e profissionais de saúde no contexto da atenção integral: revisão de literatura. Rev Bras Geriatr Gerontol [internet]. 2011 [acesso em 18 out 2019];14(1):147-57. Disponível em: http://www.scielo.br/pdf/rbgg/v14n1/a15v14n1.pdf

8. BRASIL/Ministério da Saúde. Boletim epidemiológico AIDS. 2008. Disponível em <http://www.aids.gov.br>. Acesso em 25 de março de 2013.

9. Bertoncini BZ, Moraes KS, Kulkamp IC. Comportamento sexual em adultos maiores de 50 anos infectados pelo HIV. J Bras Doenças Sex Transm [internet]. 2007 [acesso em 18 out 2019];19(2):75-9. Disponível em: http://www.dst.uff.br/revista19-2-2007/3.pdf

10. Godoy VS, Ferreira MD, Silva EC, Gir E, Canini SRMS. O perfil epidemiológico da AIDS em idosos utilizando Sistemas de Informação em Saúde do DATASUS: realidades e desafios. J Bras Doenças Sex Transm [internet]. 2008 [acesso em 18 out 2019];20(1):7-11. Disponível em: http://www.dst.uff.br/revista20-1-2008/1.pdf

11. Toledo LSG, Maciel ELN, Rodrigues LCM, Tristão-Sá R, Fregona G. Característica e tendência da AIDS entre idosos no Estado do Espírito Santo. Rev Soc Bras Med Trop [internet]. 2010 [acesso em 18 out 2019];43(3):264-7. Disponível em: http://www.scielo.br/pdf/rsbmt/v43n3/10.pdf

12. Girondi JBR, Zanatta AB, Bastiani JAN, Nothaft SS, Santos SMA. Perfil epidemiológico de idosos brasileiros que morreram por síndrome da imunodeficiência adquirida entre 1996 e 2007 Acta Paul Enferm [internet]. 2012 [acesso em 18 out 2019];25(2):302-7. Disponível em: http://www.scielo.br/pdf/ape/v25n2/a23v25n2.pdf

13. Anderson DR, Sweeney, DJ, williams, TA. Estatística aplicada à administração e economia. 2. ed. São Paulo: Editora Cengage Learning, 2007. 597 p.

14. Lazzarotto AR, Kramer AS, Hädrich M, Tonin M, Caputo $P$, Sprinz E. O conhecimento de HIV/aids na terceira idade: estudo epidemiológico no Vale do Sinos, Rio Grande do Sul, Brasil Ciênc Saúde coletiva [internet]. 2008 [acesso em 18 out 2019];13(6):1833-40. Disponível em: http:// www.scielo.br/pdf/csc/v13n6/a18v13n6.pdf

15. Cerqueira MBR, Rodrigues RN. Fatores associados à vulnerabilidade de idosos vivendo com HIV/AIDS em Belo Horizonte (MG), Brasil. Ciênc Saúde Coletiva [internet]. 2016 [acesso em 18 out 2019];21(11):3331-8. Disponível em: http://www.scielo.br/pdf/csc/v21n11/1413-8123csc-21-11-3331.pdf

16. Rocha FCV, Freitas Filho FC, Macedo Junior JA, Rosa YRD. Conhecimento dos idosos sobre HIV/AIDS. R. Interd 2013; 6(2):137-143.

17. Camargo BV, Torres TL, Biasus F. Práticas sexuais, conhecimento sobre HIV/AIDS e atitudes a respeito da relação amorosa e prevenção entre adultos com mais de 50 anos do Sul do Brasil. Liber [internet]. 2009 [acesso em 18 out 2019];15(2):171-80. Disponível em: http://www.scielo.org.pe/pdf/liber/v15n2/a11v15n2.pdf

18. Melo HMA, Leal MCC, Marques APO, Marino JG. O conhecimento sobre Aids de homens idosos e adultos jovens: um estudo sobre a percepção desta doença. Ciênc Saúde Coletiva [internet]. 2012 [acesso em 18 out 2019];17(1):43-55. Disponível em: http://www.scielo.br/ pdf/csc/v17n1/a07v17n1.pdf

19. Pereira GS, Borges CI. Conhecimento sobre HIV/AIDS de participantes de um grupo de idosos, em Anápolis-Goiás. Esc Anna Nery Rev Enferm [internet]. 2010 [acesso em 18 out 2019];14(4):720-5. Disponível em: http://www. scielo.br/pdf/ean/v14n4/v14n4a10.pdf

20. Iles D. AIDS and mosquitoes. Med J Aust. 1985; 143(10):478.

21. Zuckerman AJ. AIDS and insects. Br Med J (Clin Res Ed). 1986;292(6528):1094-5.

22. Lazzarotto A, Reichert MT, Venker C, Kramer AS, Sprinz E. HIV/aids e meia idade: avaliação do conhecimento de indivíduos da região do Vale do Sinos (RS), Brasil. 
Ciênc Saúde Coletiva [internet]. 2010 [acesso em 18 out 2019];15(Supl.1):1185-90. Disponível em: http://www.scielo.br/pdf/csc/v15s1/027.pdf

23. Costa AP, Costa PJ, Albuquerque SCO. Conhecimento de HIV/aids entre os idosos da Unidade de Saúde da Família João Pacheco Freire Filho, Arcoverde - Pernambuco. Saúde Colet Debate. 2012;2(1):9-19

24. Nardelli, GG, Malaquias BSS, Gaudenci EM, Ledic CS, Azevedo NF, Martins VE, et al. Conhecimento sobre síndrome da imunodeficiência humana de idosos de uma unidade de atenção ao idoso. Rev Gaúcha Enferm [internet]. 2016 [acesso em 18 out 2019];37(esp):e2016-0039. Disponível em: http://www.scielo.br/pdf/rgenf/v37nspe/0102-6933rgenf-1983-14472016esp2016-0039.pdf

25. Madruga MDD, Vieira KFL, Almeida SA. Fatores de vulnerabilidade dos idosos ao HIV/AIDS: uma revisão integrativa. Rev Pesqui (Univ Fed Estado Rio J, Online) [internet]. 2018 [acesso em 18 out 2019];10(3):12-8. Disponível em: http://www.seer.unirio.br/index.php/ cuidadofundamental/article/viewFile/7595/6580

26. Fernandes MGM, Silva AO, Loureiro LSN, Medeiros ACT. Indicadores e condições associadas ao envelhecimento bem-sucedido: revisão integrativa da literatura. Cogitare Enferm. 2011;16(3):545-8. 